\title{
Unilateral Central Areolar Choroidal Dystrophy
}

\author{
Rika Tsukii $^{1}$, Shinji Makino ${ }^{1 *}$
}

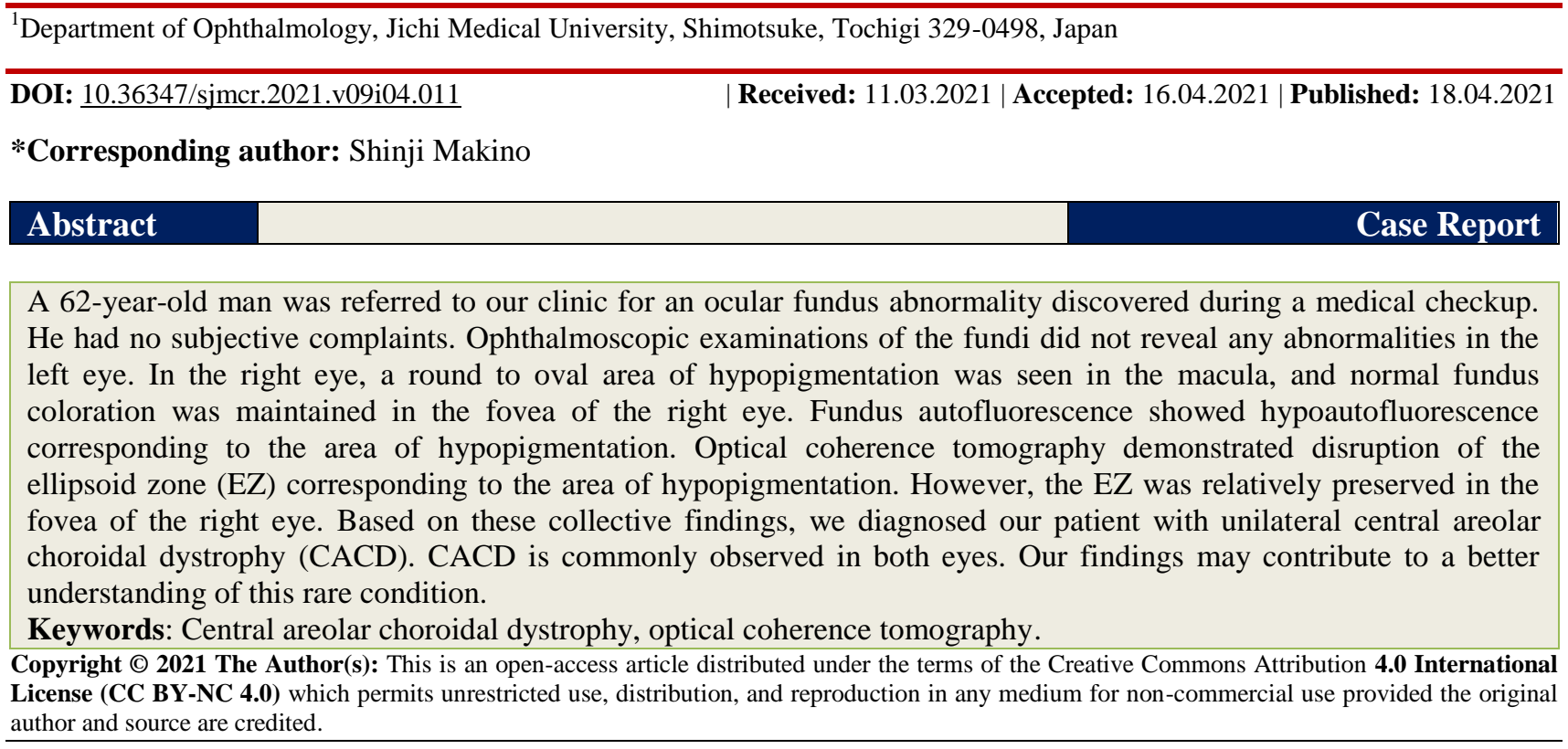

\section{INTRODUCTION}

Central areolar choroidal dystrophy (CACD) is a hereditary retinal disorder that principally affects the macula, often resulting in a well-defined area of atrophy of the retinal pigment epithelium (RPE) and choriocapillaris in the center of the macula [1-4]. Dysfunction of macular photoreceptors usually leads to a decrease in visual acuity, generally occurring between the ages of 30 and 60 years. CACD is commonly observed in both eyes. Here, we report an unusual case of unilateral CACD.

\section{Case Report}

A 62-year-old Japanese man was referred to our clinic for an ocular fundus abnormality discovered during a medical checkup. He had no subjective complaints. His medical history was unremarkable, and there was no family history of ocular disease. On ophthalmic examination, his best corrected visual acuity (BCVA) was 20/20 in the right eye and 20/20 in the left eye. Both anterior segments as well as ocular pressures were normal. Ophthalmoscopic examinations of the fundi did not reveal any abnormalities in the left eye. In the right eye, a round to oval area of hypopigmentation was seen in the macula (Figure-1), and normal fundus coloration was maintained in the fovea of the right eye.
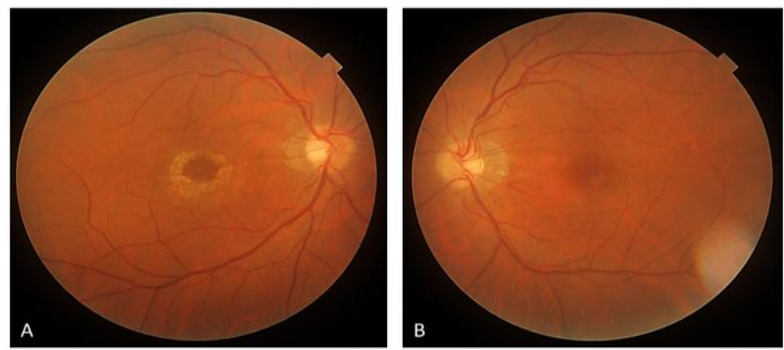

Fig-1: Fundus photographs of the (A) right and (B) left eyes

Note a round to oval area of hypopigmentation in the right eye.

Fundus autofluorescence (FAF) showed hypoautofluorescence corresponding to the area of hypopigmentation (Figure-2A).
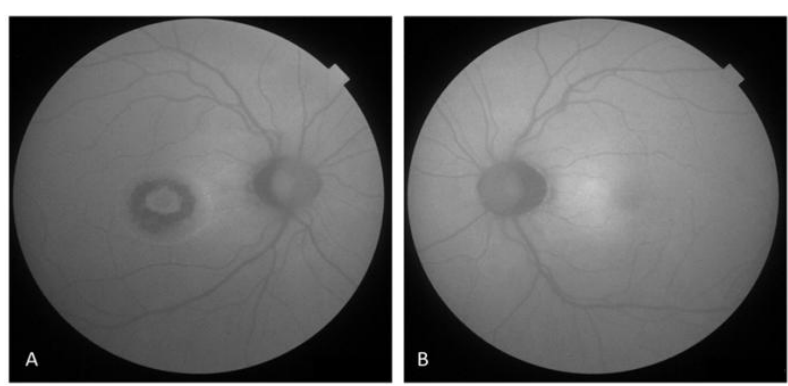

Fig-2: Fundus autofluorescence of the (A) right and (B) left eyes 
Note hypoautofluorescence corresponding to the area of hypopigmentation in the right eye.

Optical coherence tomography (OCT) demonstrated disruption of the ellipsoid zone (EZ) corresponding to the area of hypopigmentation (Figure3, arrowheads). However, the EZ was relatively preserved in the fovea of the right eye (Figure-3, arrows).

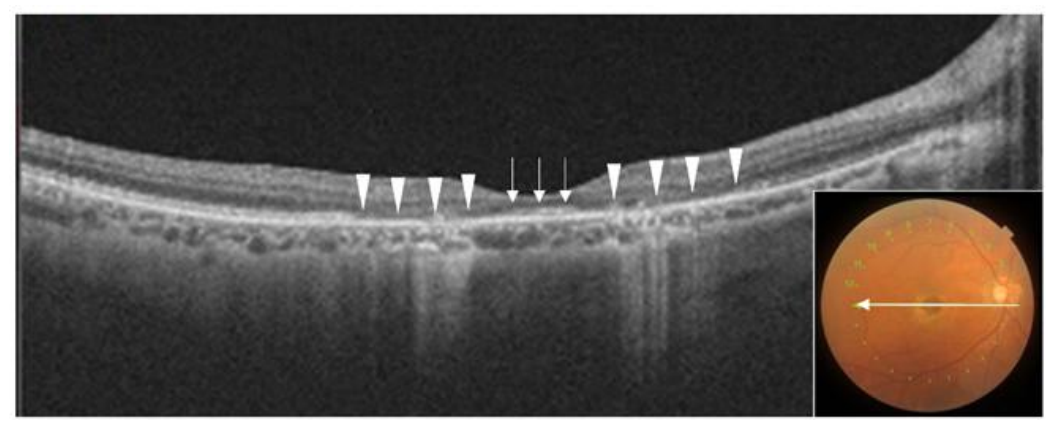

Fig-3: Optical coherence tomography images of the right eye

Note marked attenuation of the ellipsoid zone (arrowheads), while the ellipsoid zone was relatively preserved in the fovea (arrows).

Based on these collective findings, we diagnosed our patient with unilateral CACD. The patient was followed up without any treatment. Six months after his initial visit, his BCVA remained unchanged.

\section{DiscUSSION}

In this study, we present a case of unilateral CACD in a 62-year-old man. Hoyng and Deutman [1] divided the development of CACD into four stages: In stage I, slight parafoveal pigmentary changes of the RPE are seen, usually in adolescent patients. Stage II is characterized by a round to oval, mildly atrophic hypopigmented area of 1.5 to several disc diameters. In stage III, an area of well-circumscribed atrophy of RPE and choriocapillaris appear outside the fovea. In stage $\mathrm{IV}$, this well-defined chorioretinal atrophy involves the fovea. When the area of profound chorioretinal atrophy reaches the center of the macula and is about to involve central foveal function with fairly good visual acuity, the lesions are categorized as stage III-IV.

In the largest series of patients with CACD, Boon et al. [2] evaluated the clinical characteristics, follow-up data and molecular genetic background in 103 patients with this condition. According to their report, asymmetry of stages between both eyes was observed in 26 patients (25\%), and relative foveal sparing in advanced CACD, with a correspondingly good visual acuity, was seen in four patients. In addition, they described that the course of CACD was variable: some patients never reached stage IV, III, or even stage II CACD. Most patients with CACD takes more than a decade to progress from stage II to stage III. Therefore, the visual acuity varies widely, generally depending on the disease stage.
Recently, OCT imaging has provided insights into the retinal architectural changes that occur in CACD. Gocho et al., [3] described that OCT showed loss of reflectivity and disruption of the EZ and inter $\neg$ digitation zone (IZ) and thinning of the outer nuclear layer $(\mathrm{ONL})$ in the parafoveal region. The EZ, IZ, and ONL were more disrupted in the parafoveal region than in the foveal center and peripheral retina.

Autosomal-dominant CACD is genetically heterogeneous, but mutations in the peripherin/RDS gene (PRPH2 or peripherin-2) seem to be the most common cause. Boon et al., [2] evaluated molecular genetic background in 103 patients with CACD. According to their report, five different mutations in peripherin/RDS have been described in autosomaldominant CACD: p.Arg142Trp, p.Arg172Trp, p.Arg172Gln, p.Arg195Leu, and p.Leu307fsX83. The large majority of macular lesions encountered in carriers of the p.Arg142Trp mutation displayed the typical aspect of a certain stage of CACD, although with varying age at onset and rate of progression. The onset of visual disturbances is usually before the age of 40 in CACD caused by the p.Arg172Trp or p.Arg195Leu mutation in peripherin/RDS. In p.Arg142Trp-related CACD, visual symptoms usually manifest in the middle of the fifth decade of life. Our patient also had no family history and was completely asymptomatic until the age of 62 years.

\section{CONCLUSION}

Our findings are based on a single case; additional studies including long-term follow-up, additional cases, and genetic examination are necessary. Our findings may contribute to a better understanding of this rare condition.

Disclosure: The author declares no conflict of interest. 


\section{REFERENCES}

1. Hoyng CB, Deutman AF. The development of central areolar choroidal dystrophy. Graefes Arch Clin Exp Ophthalmol. 1996; 234: 87-93.

2. Boon CJ, Klevering BJ, Cremers FP, ZonneveldVrieling MN, Theelen T, Den Hollander AI, Hoyng CB. Central areolar choroidal dystrophy. Ophthalmology. 2009 Apr 1;116(4):771-82.

3. Gocho K, Akeo K, Itoh N, Kameya S, Hayashi T, Katagiri S, Gekka T, Ohkuma Y, Tsuneoka H, Takahashi H. High-resolution adaptive optics retinal image analysis at early stage central areolar choroidal dystrophy with PRPH2 mutation. Ophthalmic Surgery, Lasers and Imaging Retina. 2016 Dec 1;47(12):1115-26.

4. Bax NM, Valkenburg D, Lambertus S, Klevering BJ, Boon CJ, Holz FG, Cremers FP, Fleckenstein $\mathrm{M}$, Hoyng $\mathrm{CB}$, Lindner M. Foveal sparing in central retinal dystrophies. Investigative ophthalmology \& visual science. 2019 Aug 1;60(10):3456-67. 